\title{
Fourier-space crystallography as group cohomology
}

\author{
David A. Rabson \\ Department of Physics, PHY 114, University of South Florida, Tampa, Florida 33620 \\ Benji Fisher \\ Department of Mathematics, Boston College, Chestnut Hill, Massachusetts 02467
}

(Received 3 May 2001; revised manuscript received 27 September 2001; published 17 December 2001)

\begin{abstract}
We reformulate Fourier-space crystallography in the language of cohomology of groups. Once the problem is understood as a classification of linear functions on the lattice, restricted by a particular group relation and identified by gauge transformation, the cohomological description becomes natural. We review Fourier-space crystallography and group cohomology, quote the fact that cohomology is dual to homology, and exhibit several results, previously established for special cases or by intricate calculation, that fall immediately out of the formalism. In particular, we prove that two phase functions are gauge equivalent if and only if they agree on all their gauge-invariant integral linear combinations and show how to find all these linear combinations systematically.
\end{abstract}

DOI: 10.1103/PhysRevB.65.024201

PACS number(s): 61.50.Ah, 61.44.Br, 61.44.Fw

\section{INTRODUCTION}

The discovery in 1984 of crystals with fivefold symmetry, ${ }^{1}$ and therefore no periodicity, revived interest in Bienenstock and Ewald's 1962 reformulation of crystallography in Fourier space. ${ }^{2}$ Mermin and collaborators have applied this Fourier-space crystallography to the classification of the space groups of quasiperiodic and periodic crystals, ${ }^{3-5}$ modulated crystals, ${ }^{6}$ and color groups. ${ }^{7}$ We show here that this technique, by now familiar to crystallographers, has a particularly simple expression in the language of homological algebra.

Section II quickly reviews the basis of Fourier-space crystallography. The phase function $\Phi_{g}(\mathbf{k})$, defined for $\mathbf{k}$ in the reciprocal lattice and $g$ in the point group, facilitates the classification of space groups. We review the gauge equivalence of two phase functions. In Sec. III, we consider the necessary extinctions that occur in the diffraction pattern when $g$ leaves $\mathbf{k}$ invariant, yet $\Phi_{g}(\mathbf{k})$ does not vanish. It would be simple if two phase functions that agreed at all such pairs $(g, \mathbf{k})$ were gauge equivalent, but in fact there are two crystallographic counterexamples; however, when we consider the gauge equivalence of linear combinations of phase functions, we can state the result (Theorem 1) that two phase functions are equivalent if and only if all their gaugeequivalent linear combinations agree. Section IV reviews work by Mermin and König on the most important such linear combination, one that relates to a ray representation of the point group and necessitates an electronic degeneracy in the system. Section V places the phase function $\Phi$ in a cohomology group, while Sec. VI puts gauge-invariant linear combinations of phase functions in a related homology group. In Sec. VII, we invoke the duality of these cohomology and homology groups to prove Theorem 1 (an alternative, elementary, proof being presented in the Appendix). We also prove that for any phase function there exists a gauge in which it takes only rational values (with set denominator). Section VIII applies our results to simplifying the classification of space groups, and Sec. IX addresses the ray represen- tation of Sec. IV in homological language. Section X compares our results to a real-space treatment.

\section{FOURIER-SPACE CRYSTALLOGRAPHY}

Mermin and others have argued persuasively ${ }^{8,9}$ that the theoretical significance of quasicrystals lies not so much in relaxing the requirement of periodicity as in replacing exact identity of a density function (e.g., electronic or nuclear) under symmetry operations with indistinguishability. We will review Fourier-space crystallography only tersely, in order to establish the notation; for a more developed exposition, we refer to Ref. 10.

The reciprocal lattice $\mathcal{L}$ (or simply lattice, since there is no direct-space lattice of translations in the aperiodic cases) is the closure under addition of the set of Bragg points $\mathbf{k}$ at which the Fourier transform $\rho(\mathbf{k})$ of the direct-space density function does not vanish. Crystals, periodic or quasiperiodic, have reciprocal lattices generated as all integral linear combinations of a finite set of vectors $\mathbf{k}$; we do not consider noncrystals. A different density function $\rho^{\prime}$ is said to be indistinguishable from $\rho$ if all spatially averaged $n$-point correlation functions, representing macroscopic physical measurements, are the same for the two densities: in Fourier space,

$$
\rho^{\prime}\left(\mathbf{k}_{1}\right) \rho^{\prime}\left(\mathbf{k}_{2}\right) \cdots \rho^{\prime}\left(\mathbf{k}_{n}\right)=\rho\left(\mathbf{k}_{1}\right) \rho\left(\mathbf{k}_{2}\right) \cdots \rho\left(\mathbf{k}_{n}\right)
$$

for all lattice vectors summing to zero $\left(\Sigma \mathbf{k}_{i}=0\right)$. This holds if and only if

$$
\rho^{\prime}(\mathbf{k})=e^{2 \pi i \chi(\mathbf{k})} \rho(\mathbf{k}),
$$

where $\chi$, called a gauge function, is any real-valued linear function (taking values modulo 1 ) (Ref. 11) on $\mathcal{L}$. We are particularly interested in the case that the first density function in Eq. (2) results from acting on the second with an element $g$ of a point group, $G \subset \mathrm{O}(3)$, for then

$$
\rho^{\prime}(\mathbf{k})=\rho(g \mathbf{k})=e^{2 \pi i \Phi_{g}(\mathbf{k})} \rho(\mathbf{k}),
$$


where $\Phi_{g}$, the particular gauge function for this $g$, is called a phase function. Phase functions are constrained by the group-compatibility condition for $g, h \in G$,

$$
\Phi_{g h}(\mathbf{k})=\Phi_{g}(h \mathbf{k})+\Phi_{h}(\mathbf{k}),
$$

a consequence of the group action on the lattice.

The phase function defines algebraically the action of the point group on the density and so, together with the lattice and orientation, encodes the information of the traditionally defined space group. However, altogether too much freedom remains, for two phase functions $\Phi^{\prime}$ and $\Phi$ related by a gauge function through

$$
\Phi_{g}^{\prime}(\mathbf{k})-\Phi_{g}(\mathbf{k})=\chi(g \mathbf{k}-\mathbf{k})
$$

yield indistinguishable densities. To classify space groups, one therefore first computes all possible phase functions satisfying Eq. (4), then groups into gauge-equivalence classes phase functions differing only by a gauge, as in Eq. (5). To complete the classification, one must also consider scale invariance and the orientation of $G$ with respect to $\mathcal{L}$. Reference 10 discusses in detail the classification of Bravais classes, arithmetic crystal classes, and space-group types. In the present work, we are concerned only with equivalence classes of phase functions.

Until now, the grouping into gauge-equivalence classes has required a clever choice of specific gauge in which all but a few values of $\Phi_{g}(\mathbf{k})$ vanish; homological algebra provides a more systematic approach to the classification, for it is precisely the group of functions satisfying Eq. (4) with the identification (5) that constitutes the fundamental object of cohomology.

\section{EXTINCTIONS, AN UNTRUE PROPOSITION, AND A THEOREM}

The class containing the trivial $\Phi$, for which all values $\Phi_{g}(\mathbf{k})$ vanish, is called symmorphic. Perhaps the most direct result of Fourier-space crystallography concerns necessary extinctions in the diffraction patterns of materials with certain nonsymmorphic space groups. The simplest example of the $n$-point correlation function in Eq. (1), $\rho(\mathbf{k}) \rho(-\mathbf{k})$ $=|\rho(\mathbf{k})|^{2}$, is proportional to the intensity in a diffraction experiment. As is well known, materials with certain space groups show zero intensity at particular lattice points $\mathbf{k}$. From the Fourier-space point of view, if some point-group element $g$ leaves $\mathbf{k}$ invariant and if $\Phi_{g}(\mathbf{k}) \neq 0$, Eq. (3) requires that $\rho(\mathbf{k})$ itself should vanish.

By Eq. (5), the condition $g \mathbf{k}=\mathbf{k}$ means that all specimens $\Phi$ in a particular equivalence class of phase functions will take the same value for that choice of $g$ and $\mathbf{k}$, regardless of gauge. We call the particular value $\Phi_{g}(\mathbf{k})$ a gauge-invariant part.

By definition, if two phase functions are related by a gauge (5), then they agree on all their gauge-invariant parts, but the converse is not true: out of the 230 periodic space groups in three dimensions, there are precisely two nonsymmorphic space groups with no nonzero gauge-invariant $\Phi_{g}(\mathbf{k})$. Thus they agree with their symmorphic cousins whenever $g \mathbf{k}=\mathbf{k}$, yet they are not symmorphic. Lacking nonzero gauge-invariant $\Phi_{g}(\mathbf{k})$, the two space groups $I 2{ }_{1} 2_{1} 2_{1}$ and $I 2{ }_{1} 3$ exhibit no systematic extinctions. For the other 228 periodic space groups and for all known quasiperiodic space groups, it happens to be the case that two phase functions are related by a gauge if and only if they agree on all their gauge-invariant parts of the form $\Phi_{g}(\mathbf{k})$. If we knew this to be true in advance, we could use it to simplify the classification of equivalence classes of phase functions by just finding all gauge-invariant parts compatible with Eq. (4). Absent this proposition, the gauge-invariant parts are insufficient: we must find a clever choice of gauge in which all noninvariant phases vanish.

For the two peculiar cases in which one cannot make all noninvariant phases vanish, Mermin has constructed a gauge-invariant linear combination of two phase functions,

$$
\Phi_{g}\left(\mathbf{k}_{h}\right)-\Phi_{h}\left(\mathbf{k}_{g}\right),
$$

for a specific choice of commuting point-group elements $g$ and $h$ and lattice vectors $\mathbf{k}_{h}$ and $\mathbf{k}_{g}$, where neither $\Phi_{g}\left(\mathbf{k}_{h}\right)$ nor $\Phi_{h}\left(\mathbf{k}_{g}\right)$ is gauge invariant. By showing that the groupcompatibility condition (4) permits the gauge invariant (6) to assume a value either 0 (symmorphic) or $1 / 2$ (nonsymmorphic), he derives the existence of the two nonsymmorphic space groups $I 2_{1} 2_{1} 2_{1}$ for point group 222 on the orthorhombic $I$ lattice and $I 2{ }_{1} 3$ for point group 23 on the cubic $I$ lattice.

The fact that a gauge invariant of the form $\Phi_{g}(\mathbf{k})$ is simply a linear combination of one phase, while Eq. (6) is a linear combination of two, suggests a generalization of the untrue converse about gauge-invariant single phases.

Theorem 1. Two phase functions are related by a gauge if and only if they agree on all their gauge-invariant linear combinations, of the form $\Sigma_{i} \Phi_{g_{i}}\left(\mathbf{k}_{i}\right)$.

We will show (Sec. VI) that an appropriate homology group systematically classifies all gauge invariants and (Sec. VII) that its duality to a cohomology group proves the theorem. Because the proof of Theorem 1 does not rely on the specific generating relations of the lattice, it applies equally to crystals, quasicrystals, and modulated aperiodic crystals as well as to "standard" and "nonstandard" lattices. ${ }^{12}$ While nonstandard lattices have not yet been identified in nature, observations of high-symmetry aperiodic lattices in liquid crystals $^{13,14}$ and plasmas ${ }^{15}$ suggest that our results may find application.

\section{NECESSARY DEGENERACIES}

Although a nonzero value of the invariant combination (6) implies no necessary extinctions in the diffraction pattern, König and Mermin ${ }^{16}$ have given it a definite physical interpretation. They consider a reciprocal-space vector $\mathbf{q}$, not necessarily in $\mathcal{L}$, and the associated little group $G_{\mathbf{q}} \subseteq G$ consisting of elements $g$ such that

$$
\mathbf{k}_{g} \equiv \mathbf{q}-g \mathbf{q}
$$

is in the lattice. ${ }^{17}$ The Hamiltonian $h_{\mathbf{q}}$ of the electronic problem at Bloch wave vector $\mathbf{q}$ describes all bands at $\mathbf{q}$; bands 
may repel or cross. If for commuting $g$ and $h$ in $G_{\mathbf{q}}$ the invariant (6) does not vanish, every level must be degenerate with some other level at $\mathbf{q}$, so bands cross at least in pairs. We outline the argument first because the gauge invariant (6) falls out of the first-homology calculation below and second because it relates to a second cohomology group.

König and Mermin expand the Bloch wave in reciprocallattice vectors $\mathbf{k}$ and express the action of $g$ in the point group on $\mathbf{k}$ in terms of the unitary operator $U(g)$, with

$$
U(g)|\mathbf{k}\rangle=e^{2 \pi i \Phi_{g}(\mathbf{k})}\left|g \mathbf{k}-\mathbf{k}_{g}\right\rangle .
$$

The operators $U(g)$ commute with the Hamiltonian $h_{\mathbf{q}}$ and constitute a ray representation of $G_{\mathbf{q}}$, for

$$
U(g) U(h)=e^{-2 \pi i \Phi_{g}\left(\mathbf{k}_{h}\right)} U(g h) .
$$

The map

$$
\lambda(g, h)=e^{-2 \pi i \Phi_{g}\left(\mathbf{k}_{h}\right)}
$$

from $G \times G$ to the unit circle is called the factor system at $\mathbf{q}$.

Suppose that $E$ is a nondegenerate energy level of $h_{\mathbf{q}}$, and let $|\psi\rangle$ be the corresponding eigenvector. Since the Hamiltonian commutes with all the $U(g),|\psi\rangle$ must also be an eigenvector of each, so

$$
U(g) U(h)|\psi\rangle=U(h) U(g)|\psi\rangle .
$$

Comparing this commutation of matrices $U$ to the ray representation (9), König and Mermin find, for commuting $g$ and $h$, that Eq. (6) must vanish. If it does not, every band must cross at least one other band at $\mathbf{q}$.

\section{GAUGE-EQUIVALENCE CLASSES AND THE FIRST COHOMOLOGY GROUP}

Brown ${ }^{18}$ traces the theory of group cohomology to a 1904 work by Schur $^{19}$ on ray representations such as Eq. (9). We now use this theory to describe the classification of phase functions up to gauge equivalence. ${ }^{20} \mathrm{In} \mathrm{Sec}$. X, we compare this Fourier-space cohomology to group cohomology in realspace crystallography.

The phase function $\Phi_{g}$, which maps the lattice $\mathcal{L}$ to the real numbers modulo unity, has three defining characteristics: (i) it is linear, (ii) it satisfies the group-compatibility condition (4), and (iii) it is defined only modulo gauge functions of the form (5). Cohomology conveniently packages functions of just such description.

The map $\Phi$, which takes $g \in G$ to $\Phi_{g}$, is an example of a 1-cochain, the set of all of which we call $C^{1}$. We denote by $\mathcal{L}^{*}=\operatorname{Hom}(\mathcal{L}, \mathrm{R} / \mathrm{Z})$ the group of all real-valued $(\bmod 1)$ linear functions on the lattice $\mathcal{L}$; the phase function $\Phi_{g}$ is in $\mathcal{L}^{*}{ }^{21}$ More generally, an $n$-cochain in $C^{n}$ is a function that maps $n$ group elements from $G$ to $\mathcal{L}^{*}$. Here $C^{n}$ has a group structure under addition. The gauge $\chi$, taking no point-group arguments, is a 0 -cochain.

Connecting these functions is a coboundary map, $d: C^{n}$ $\rightarrow C^{n+1}$, such that two maps in a row yield zero identically, $d^{(n+1)} \circ d^{(n)}=0$. Applied specifically to the lowest-order cases, ${ }^{22}$

$$
(d \chi)(g)=\chi \circ g-\chi
$$

and

$$
(d \Phi)(g, h)=\Phi_{g} \circ h-\Phi_{g h}+\Phi_{h} .
$$

Equation (12) identifies those 1-cochains that are coboundaries of 0 -cochains as the group of gauge transformations [by Eq. (5)]; we define $B^{n}=\operatorname{im}\left(C^{n-1} \stackrel{d}{\rightarrow} C^{n}\right)$ as the set of all $n$-coboundaries. Similarly, the right-hand side of Eq. (13) must vanish if $\Phi$ is to satisfy the group-compatibility relation (4); we define $Z^{n}=\operatorname{ker}\left(C^{n} \stackrel{d}{\rightarrow} C^{n+1}\right)$ as the set of all $n$-cocycles: that is, $n$-cochains with vanishing coboundaries.

The quotient group $H^{1}=Z^{1} / B^{1}$, or cocycles modulo coboundaries, is called the first cohomology group of $G$ with coefficients in $\mathcal{L}^{*}$. Membership in $Z^{1}$ establishes group compatibility, while modding out by $B^{1}$ identifies functions differing only by gauge transformation, so $H^{1}$ is exactly the group of equivalence classes of phase functions.

\section{INVARIANTS AND THE FIRST HOMOLOGY GROUP}

To prove Theorem 1, we must find all gauge-invariant linear combinations of phase functions. Suppose the linear function $f: H^{1} \rightarrow \mathbb{R} / Z$ takes an equivalence class of phase functions to a real number; we may think of any gauge invariant in these terms. Since the value of a gauge invariant is the same for any member $\Phi$ of an equivalence class $\{\Phi\}$, we may write $f(\{\Phi\})=f(\Phi)$ as only a slight abuse of notation. We may turn this around to think of $\Phi$ mapping $f$ to a number.

To produce a number in the unit interval, a cochain acts on integral linear combinations of ordered sets each containing one lattice vector and $n$ group elements, which we can write (following Brown's convention ${ }^{18}$ ) $\sum_{i} \mathbf{k}_{i}\left[g_{1 i}\left|g_{2 i}\right| \cdots \mid g_{n i}\right]$ for $\mathbf{k}_{i} \in \mathcal{L}$ and $g_{j i} \in G$. Since $\mathcal{L}$ already absorbs any integral coefficients, we need not write them explicitly. Such linear combinations are called $n$-chains and the set of all $n$-chains $C^{n}=\mathcal{L}\left[G^{n}\right]$.

A cochain $\Phi$ in $C^{1}$ and a chain $c=\sum_{i} \mathbf{k}_{i}\left[g_{i}\right]$ in $C_{1}$ act on each other through the bracket

$$
\langle\Phi, c\rangle=\sum_{i} \Phi_{g_{i}}\left(\mathbf{k}_{i}\right) \in \mathbb{R} / Z
$$

We let $C_{1}$ inherit the additive group structure of $\mathcal{L}: \mathbf{k}\left[g_{1}\right]$ $+\mathbf{k}^{\prime}\left[g_{1}\right]=\left(\mathbf{k}+\mathbf{k}^{\prime}\right)\left[g_{1}\right]$.

By direct analogy with the coboundary operator in Eqs. (12) and (13), we define a boundary map $\partial: C_{n} \rightarrow C_{n-1}$ such that two maps in a row yield zero. Note that $\partial$ decrements the number of copies of $G$ in the chain, whereas the $d$ operator on cochains increments it. In the lowest-order cases, ${ }^{23}$

$$
\partial \mathbf{k}[g]=g \mathbf{k}-\mathbf{k}
$$

and

$$
\partial \mathbf{k}[g \mid h]=(h \mathbf{k})[g]-\mathbf{k}[g h]+\mathbf{k}[h] .
$$


1-cycles are chains $c=\sum_{i} \mathbf{k}_{i}\left[g_{i}\right]$ satisfying

$$
\partial c=\sum_{i}\left(g_{i} \mathbf{k}_{i}-\mathbf{k}_{i}\right)=0
$$

Let $Z_{1}=\operatorname{ker}\left(C_{1} \stackrel{\partial}{\rightarrow} C_{0}\right)$ denote the set of all 1-cycles. Applying the bracket (14) to $c$ satisfying Eq. (17), we find that 1-cycles give gauge-invariant linear combinations of phases, and since the condition for gauge invariance of a general linear combination of phases $\sum_{i} \Phi_{g_{i}}\left(\mathbf{k}_{i}\right)$ is, by Eq. (5), precisely Eq. (17), all such gauge-invariant linear combinations come from 1-cycles. In the case that $c=\mathbf{k}[g]$ in Eq. (17), the gauge invariant takes the form $\Phi_{g}(\mathbf{k})$ for $g \mathbf{k}=\mathbf{k}$, but in general a linear combination, such as Eq. (6), must be allowed.

Similarly, 1-boundaries are those 1-chains that can be written as boundaries of 2-chains, as in Eq. (16) or integral linear combinations thereof: $B_{1}=\operatorname{im}\left(C_{2} \rightarrow C_{1}\right)$. Because $\partial^{\circ} \partial=0$, every 1 -boundary is a 1-cycle, representing a gauge invariant. Again applying the bracket we discover that the phases at boundaries (16) are necessarily zero by the group-compatibility condition (4). Thus, although 1-boundaries are gauge invariants, they are trivial. We mod them out in defining the first homology group of $G$ with coefficients in $\mathcal{L}$ :

$$
H_{1}=Z_{1} / B_{1} .
$$

First homology is the group of nontrivial gauge invariants.

We have already commented that a real-valued function $f$ on $H^{1}$ is unaffected by gauge (i.e., by the addition of a coboundary to a cocyle $\Phi$ ) and that the addition of a boundary to a cycle $x$ does not change any phase. These facts establish that the bracket, which we introduced in Eq. (14) as a function $C^{1} \times C_{1} \rightarrow \mathrm{R} / \mathbb{Z}$, is in fact well defined on $H^{1} \times H_{1}$, which we may express concisely thus:

$$
\langle\Phi+d \chi, x+\partial y\rangle=\langle\Phi, x\rangle,
$$

where $\Phi$ is a phase function $(d \Phi=0), d \chi$ a gauge transformation of the form $(5), x \in C_{1}$ a cycle $(\partial x=0)$, and $\partial y$ a boundary, $y \in C_{2}$.

\section{DUALITY AND THE PROOF OF THEOREM 1}

We note a striking similarity between $H^{1}$ and $H_{1}$. While the former contains all linear functions satisfying group compatibility, identifying those related by gauge transformation, the latter contains all linear gauge invariants, identifying any whose difference is made trivial by group compatibility. In fact, homological algebra affirms their duality. Before stating this central result, we note the following. ${ }^{24}$

Lemma 2. For $G$ a finite group with $|G|$ elements and $\Phi$ $\in H^{1},|G| \Phi=0$ (where $0 \in H^{1}$ denotes the set of phase functions gauge equivalent to zero). In other words, for any phase function $\Phi$, there exists a gauge in which every $\Phi_{g}(\mathbf{k})$ is rational with denominator $|G|$.

This is quite plausible, since the only constraints on phases are those imposed by group compatibility (4) applied through the constituent relations of the point group. Specifi- cally, summing over $g$ in Eq. (4) and noting that $\Sigma_{g} \Phi_{g h}$ $=\Sigma_{g} \Phi_{g}$, we find

$$
|G| \Phi_{h}(\mathbf{k})=\chi(h \mathbf{k}-\mathbf{k}),
$$

where $\chi(\mathbf{k})=-\Sigma_{g} \Phi_{g}(\mathbf{k})$ clearly satisfies the definition of a gauge function. Then $|G|$ times any phase function is a pure gauge, proving the lemma. Note that we may not divide by $|G|$ to show that all phase functions are gauge equivalent to zero, because $\chi$ is defined only modulo unity.

Having found a gauge in which a phase function takes rational values, we define $\mathcal{L}^{\prime}=\operatorname{Hom}(\mathcal{L}, \mathrm{Q} / \mathrm{Z}) \subseteq \mathcal{L}^{*}$ and quote the following standard result from cohomology. ${ }^{25}$

Lemma 3. For $G$ a finite group and $\mathcal{L}$ a free Abelian group, the groups $H^{n}\left(G, \mathcal{L}^{\prime}\right)$ and $H_{n}(G, \mathcal{L})$ are dual through the bracket (14) $\langle\rangle:, \quad H^{n} \times H_{n} \rightarrow \mathrm{Q} / \mathrm{Z}$.

Duality means that if $X$ serves as a minimal basis for $H_{1}$, there exists a basis $\left\{\Phi^{x}\right\}$ for $H^{1}$ labeled by $x \in X$ and that any $\Phi \in H^{1}$ can be decomposed uniquely as a linear combination of the basis elements $\Phi^{x}$ with integral coefficients equal to $\langle\Phi, x\rangle /\left\langle\Phi^{x}, x\right\rangle$. Therefore, if we know the value $\langle\Phi, x\rangle$ of a phase function on each of the nontrivial gauge invariants $x$ spanning $H_{1}$, by linearity we know $\Phi$ up to gauge transformation. This proves Theorem 1.

We give in the Appendix an alternative, elementary proof of Theorem 1 by constructing the gauge function relating two phase functions that agree on all gauge-invariant linear combinations.

\section{CALCULATING SPACE GROUPS (EXAMPLE)}

Homological algebra offers a powerful tool for systematically finding all gauge invariants. By Theorem 1, it furthermore frees us from having to specify a gauge: once we have the gauge invariants, we no longer have to prove on a case-by-case basis that all other phases can be made to vanish. As an example, we compute the two possible spacegroup classes on the body-centered orthorhombic directspace lattice (which is face centered in reciprocal space) with the point group 222. Instead of having to hunt for the gauge invariant (6), we shall see it fall out of the formalism naturally.

The calculation is straightforward. First, we calculate the group $Z_{1}$ of gauge invariants by acting with the boundary operator $\partial$ on 1-chains $\mathbf{k}[g]$. We then wish to remove from the list all boundaries in $B_{1}$, since these represent the "trivial" consequence of group compatibility (4); to do so, we let $\partial$ act on 2-chains $\mathbf{k}[g \mid h]$. After this elimination, we find a homology group with only two elements. The entire procedure is easily automated; for example, the NullSpace[] and LatticeReduce[] functions of Wolfram's Mathematica program can be used to find minimal bases over $\mathbb{Z}$ for $Z_{1}$ and $B_{1}$.

Let $\hat{\mathbf{e}}^{i}, i=1,2,3$, constitute the usual Cartesian axes. We name the three sides of the conventional reciprocal cell $\mathbf{x}$ $=a \hat{\mathbf{e}}^{1}, \mathbf{y}=b \hat{\mathbf{e}}^{2}$, and $\mathbf{z}=c \hat{\mathbf{e}}^{3}$, where $a, b$, and $c$ are all different, and generate the face-centered reciprocal lattice $\mathcal{L}$ (dual to the body-centered direct lattice) with the vectors $b_{1}$ $=\frac{1}{2}(011), b_{2}=\frac{1}{2}(101)$, and $b_{3}=\frac{1}{2}(110)$ [all in terms of the 
$(\mathbf{x}, \mathbf{y}, \mathbf{z})$ basis]. The point group is $G=\left\{e, d_{1}, d_{2}, d_{3}\right\}$, where $d_{j}$ leaves $\hat{\mathbf{e}}^{j}$ invariant, $d_{1} d_{2}=d_{3}$ et cycl., and $d_{i}^{2}=e$, the identity. $G$ is generated by $d_{1}$ and $d_{2}$.

To calculate the group of gauge invariants, $Z_{1}$, we apply $\partial$ to the (additive) generators of 1 -chains. The chains $\mathbf{k}[e]$ are cycles, since $e$ leaves all vectors invariant. We can show that they are also boundaries, for by group compatibility,

$$
\mathbf{k}[g h] \doteq(h \mathbf{k})[g]+\mathbf{k}[h],
$$

where $\doteq$ denotes equality up to the boundary $\partial \mathbf{k}[g \mid h]$. Setting $g=h=e$, we then have $\mathbf{k}[e] \doteq 0$, so we leave it out of the computation. Equation (21) furthermore tells us that we need consider only the generating elements $d_{1}$ and $d_{2}$, not $d_{3}=d_{1} d_{2}$.

One easily verifies that

$$
\left(\partial \mathbf{b}_{i}\left[d_{j}\right]\right)_{k}=\left|\epsilon_{i j k}\right|-\delta_{i k}-\delta_{j k},
$$

where the subscript $k$ indicates the resulting lattice component in the $\left(\mathbf{b}_{1}, \mathbf{b}_{2}, \mathbf{b}_{3}\right)$ basis, $\delta$ is the Kronecker delta, and $\boldsymbol{\epsilon}$ the totally antisymmetric tensor.

With $i=1,2,3$ and $j$ restricted to 1,2 , we can think of the left-hand side of Eq. (22) as a $3 \times 6$ matrix whose threedimensional null space over the integers is $Z_{1}$ (aside from the boundaries we have already eliminated). It is a standard result of linear algebra that row reduction without division yields a primitive basis over the integers; ${ }^{26}$ we find for this basis

$$
\begin{gathered}
z_{1}=\mathbf{x}\left[d_{1}\right], \\
z_{2}=\mathbf{y}\left[d_{2}\right], \\
z_{3}=-\mathbf{b}_{1}\left[d_{2}\right]+\mathbf{b}_{2}\left[d_{1}\right] .
\end{gathered}
$$

We now know all the gauge invariants and seek, by computing $B_{1}$, to identify those that are related by group compatibility (4).

To find the 1-boundaries not already identified, we must apply $\partial$ to the 27 two-chains,

$$
\mathbf{b}_{i}\left[d_{j} \mid d_{k}\right] ;
$$

this number is reduced to 18 if we notice that $\mathbf{k}\left[g \mid h_{1} h_{2}\right]$ has the same boundary as $\left(h_{2} \mathbf{k}\right)\left[g \mid h_{1}\right]+\mathbf{k}\left[g h_{1} \mid h_{2}\right]-\mathbf{k}\left[h_{1} \mid h_{2}\right]$, so that in Eq. (24) we may restrict $k$ to $1,2$.

After eliminating duplications and zeros, we get seven boundaries. Rearranging the rows into echelon form, we find only three that are linearly independent: $z_{1}, z_{2}$, and $2 z_{3}$ are all boundaries. This identifies all even multiples of the cycle $z_{3}$ in $H_{1}$, which therefore contains just a single nonzero invariant:

$$
H_{1}(G, \mathcal{L})=\left\{0, z_{3}\right\} .
$$

Since $2 z_{3} \doteq 0, \Phi\left(z_{3}\right)$ can take values only 0 and $1 / 2$.

The invariant $z_{3}$ is precisely the linear combination (6) of phases used in Ref. 5 to distinguish the symmorphic $\left[I 222, \Phi\left(z_{3}\right)=0\right]$ from the nonsymmorphic $\left[I 2_{1} 2_{1} 2_{1}\right.$, $\left.\Phi\left(z_{3}\right)=1 / 2\right]$ space group. Mermin's rather shorter calculation rests on a clever choice of gauge ensuring $\Phi_{d_{1}}\left(\mathbf{b}_{3}\right)$
$=\Phi_{d_{2}}\left(\mathbf{b}_{1}\right)=\Phi_{d_{3}}\left(\mathbf{b}_{2}\right)=0$; in our comparatively pedestrian approach, the list of phases that can be discarded is inherent in the calculation of the boundaries $B_{1}$. Significantly, the present calculation is easy to automate, requiring no "judicious choice" of generators and gauges, while verification that one gets "a representative of every class" 5 is built in to the procedure. We lose the elegance of Mermin's (equivalent) calculation, but we hope we make up for some of the loss in the compact and general statement of Theorem 1 as proved in Sec. VII. We have generalized and applied essentially the ideas demonstrated in this section to the simultaneous computation of a large number of cases, including nonstandard and modulated lattices. ${ }^{27}$

\section{SECOND COHOMOLOGY AND THE FACTOR SYSTEM}

In general, the cohomology group $H^{n}(\mathfrak{G}, \mathfrak{M})$ consists of cocycles from $n$ copies of a group $\mathfrak{G}$ to a module $\mathfrak{M}$ with the identification of coboundaries. Having classified the phase functions in $H^{1}$ with $\mathfrak{G}=G$ and $\mathfrak{M}=\mathcal{L}^{\prime}$, we can calculate the factor system (10). However, the factor system also has a cohomological existence in its own right, with a different group $\mathfrak{G}$ and different module $\mathfrak{M}$. Consider

$$
\Lambda(g, h)=-(\ln \lambda) /(2 \pi i)=\Phi_{g}\left(\mathbf{k}_{h}\right),
$$

taking values in the module $\mathfrak{M}=[0,1)=R / Z$. From here on, we will use the term "factor system" to refer to $\Lambda$ rather than to $\lambda$. Since $g$ and $h$ reside in the little group $\mathfrak{G}=G_{\mathbf{q}}, \Lambda$ is a 2-cochain in $C^{2}\left(G_{\mathbf{q}}, \mathbb{R} / Z\right)$. The action of $g \in G_{\mathbf{q}}$ on $x$ in the module $\mathrm{R} / \mathrm{Z}$ is trivial: $x g=x$.

We can again impose group compatibility as a cocycle condition:

$$
\begin{aligned}
(d \Lambda)\left(g_{1}, g_{2}, g_{3}\right) \equiv & \Lambda\left(g_{1}, g_{2}\right)-\Lambda\left(g_{2}, g_{2} g_{3}\right)+\Lambda\left(g_{1} g_{2}, g_{3}\right) \\
& -\Lambda\left(g_{2}, g_{3}\right)=0 .
\end{aligned}
$$

[The equivalence to group compatibility follows from the definitions (7) and (26). $\left.{ }^{28}\right]$

If two ray representations (8) differ by a quantum phase $e^{2 \pi i \sigma(g)} \quad$ (one for each $g \in G_{\mathbf{q}}$ ) so that $U^{\prime}(g)$ $=e^{2 \pi i \sigma(g)} U(g)$, the corresponding factor systems differ by the coboundary of $\sigma$ :

$$
\Lambda(g, h)-\Lambda^{\prime}(g, h)=(d \sigma)(g, h) \equiv \sigma(g)-\sigma(g h)+\sigma(h) .
$$

Since the quantum phase has no physical consequence, we identify ray representations related by Eq. (28). The set of phase-equivalence classes of ray representations is the second cohomology group, $H^{2}\left(G_{\mathbf{q}}, \mathbb{R} / Z\right)=Z^{2} / B^{2}$.

As a final application of group cohomology, we consider a lattice vector $\mathbf{k}$ left invariant by a point-group element $g$ $\in G$. Letting $\mathfrak{G}=\langle g\rangle=\left\{g^{n} \mid n \in \mathbb{Z}\right\}$, the cyclic group generated by $g$, and $\mathfrak{M}=\mathcal{L}$, we express $\mathbf{k} \in \mathcal{L}$ as a 0 -cochain. The cocycle condition $(d \mathbf{k})\left(g^{n}\right) \equiv g^{n} \mathbf{k}-\mathbf{k}=0$ expresses the requirement that $\mathbf{k}$ be fixed by $g$. Since there are no $(-1)$ cochains, there is no identification of coboundaries, and the cohomology $H^{0}=Z^{0}$ is the group of all $\mathbf{k}$ left invariant by $g$. 


\section{COMPARISON TO REAL-SPACE COHOMOLOGY}

It has long been known that cohomology groups can be used to classify the space groups of periodic crystals; ${ }^{24,29}$ Hiller gives a particularly approachable exposition. ${ }^{30} \mathrm{We}$ now compare our formulation, using the Fourier-space lattice $\mathcal{L}$, to the traditional approach, described in terms of the periodic lattice $L$ in real space. In terms of $L$, we can describe $\mathcal{L}$ as the lattice of integer-valued homomorphisms on $L, \mathcal{L}$ $=\operatorname{Hom}(L, \mathbb{Z})$. (For mathematical convenience and to allow direct comparison with Hiller, we have in this section absorbed the conventional factor of $2 \pi$ into $\mathcal{L}$.) In the threedimensional case, if we choose a basis $\mathbf{a}_{1}, \mathbf{a}_{2}, \mathbf{a}_{3}$ of $L$, then we can describe $\mathcal{L}$ in terms of the dual basis $\mathbf{k}_{1}, \mathbf{k}_{2}, \mathbf{k}_{3}$, where $\left\langle\mathbf{k}_{i} \mid \mathbf{a}_{j}\right\rangle=\delta_{i j}$ (Kronecker delta).

Any real-valued linear form on $\mathcal{L}$ has the form $\langle\mathbf{k} \mid \mathbf{x}\rangle$ for a fixed vector $\mathbf{x}$ in real space. Recall that our phase functions $\Phi_{g}$ take values in $\mathbb{R} / \mathbb{Z}$, not $R$ : that is, we identify $\Phi_{g}(\mathbf{k})$ with $\Phi_{g}(\mathbf{k})+n$, for any integer $n$. This corresponds to identifying $\mathbf{x}$ with $\mathbf{x}+\mathbf{a}$, for any $\mathbf{a} \in L$. Thus there is a one-to-one correspondence between phase functions and elements of the quotient group $\mathbb{R}^{3} / L$, and we can write $\Phi_{g}(\mathbf{k})=\left\langle\mathbf{k} \mid \mathbf{x}_{g}\right\rangle$ for some real-space vector $\mathbf{x}_{g}$, identified with any of its translates by lattice vectors.

In Fourier-space crystallography, the phase functions $\Phi_{g}$ are required to satisfy the group-compatibility condition (4) or, equivalently, the cocycle condition $(d \Phi)(g, h)=0$ [cf. Eq. (13)]. In terms of the vectors $\mathbf{x}_{g}$ in real space, this condition becomes $\left\langle\mathbf{k} \mid \mathbf{x}_{g h}\right\rangle=\left\langle h \mathbf{k} \mid \mathbf{x}_{g}\right\rangle+\left\langle\mathbf{k} \mid \mathbf{x}_{h}\right\rangle$. Recall what it means to let $h$ (an orthogonal transformation on real space) act on $\mathbf{k}$ (a vector in Fourier space): by definition, $\langle h \mathbf{k} \mid \mathbf{x}\rangle$ $=\left\langle\mathbf{k} \mid h^{-1} \mathbf{x}\right\rangle$. Thus the group-compatibility condition becomes

$$
\mathbf{x}_{g h}=h^{-1} \mathbf{x}_{g}+\mathbf{x}_{h} .
$$

In order to compare this with the notation of Ref. 30, let $s(g)=\mathbf{x}_{g-1}$ and replace $g$ and $h$ with $g^{-1}$ and $h^{-1}$, respectively:

$$
s(h g)=\mathbf{x}_{g^{-1} h^{-1}}=\mathbf{x}_{h^{-1}}+h \mathbf{x}_{g^{-1}}=s(h)+h s(g) .
$$

This is exactly the cocycle condition used in Ref. 30, Proposition 5.1 .

Finally, consider gauge equivalence: we identify phase functions $\Phi_{g}$ and $\Phi_{g}^{\prime}$ related by a gauge transformation as in Eq. (5). In the periodic case we are considering, the gauge function can be described by $\chi(\mathbf{k})=\langle\mathbf{k} \mid \mathbf{y}\rangle$, where again $\mathbf{y}$ is a real-space vector considered modulo $L$. If the second phase function $\Phi_{g}^{\prime}$ corresponds to $\mathbf{x}_{g}^{\prime}$ in real space, then the condition (5) can now be expressed as $\left\langle\mathbf{k} \mid \mathbf{x}_{g}^{\prime}\right\rangle-\left\langle\mathbf{k} \mid \mathbf{x}_{g}\right\rangle=$ $\langle g \mathbf{k}-\mathbf{k} \mid \mathbf{y}\rangle$ or $\mathbf{x}_{g}^{\prime}-\mathbf{x}_{g}=g^{-1} \mathbf{y}-\mathbf{y}$. In terms of $s(g)=\mathbf{x}_{g}-1$ and $s^{\prime}(g)=\mathbf{x}_{g-1}^{\prime}$, this becomes

$$
s(g)-s^{\prime}(g)=\mathbf{y}-g \mathbf{y},
$$

which is exactly the coboundary condition in Ref. 30 .

In conclusion, the cohomology group $H^{1}=Z^{1} / B^{1}$ that we consider here agrees, in the periodic case, with the cohomology group $H^{1}\left(G, \mathbb{R}^{3} / L\right)$ described in Ref. 30 .

\section{ACKNOWLEDGMENT}

D. R. gratefully acknowledges the hospitality of the mathematics department at Boston College, where some of this work was done.

\section{APPENDIX: ELEMENTARY PROOF OF THEOREM 1}

We have already shown that duality proves Theorem 1, which we may restate: a phase function is gauge equivalent to zero if and only if it vanishes on all gauge-invariant linear combinations or, equivalently, a 1-cocycle is a coboundary iff it vanishes on all cycles. The implication ("only if") statements come immediately, so we prove the converse.

Although standard treatises on homological algebra prove duality of $H^{n}$ and $H_{n}$ (Lemma 3), the following elementary demonstration of Theorem 1 also explicitly constructs the gauge transformation $\chi$ for which $\Phi_{g}(\mathbf{k})=\chi(g \mathbf{k}-\mathbf{k})$ if $\Phi$ vanishes on all gauge invariants. The proof extends familiar ideas from the linear algebra of vector spaces to that of free modules (over $\mathbb{Z}$ ).

Define the linear function $\tilde{\chi}$ on all 0-boundaries $\partial c \in B_{0}$ $\left(c \in C_{1}\right)$ by

$$
\langle\widetilde{\chi}, \partial c\rangle \equiv\langle\Phi, c\rangle
$$

We can do so consistent with linearity because, by hypothesis, $\langle\Phi, z\rangle=0$ for all gauge invariants $z \in \operatorname{ker} \partial$. As a special case of Eq. (A1), for $c=\mathbf{k}[g]$, we have $\Phi_{g}(\mathbf{k})=\tilde{\chi}(g \mathbf{k}$ $-\mathbf{k})$, which almost makes $\Phi$ gauge equivalent to zero. However, to establish that $\Phi$ is purely a gauge (thus proving the theorem), we must extend $\tilde{\chi}$, which we have so far defined only on $B_{0}$, to a linear function $\chi$ defined on all $\mathcal{L}=C_{0}$. To extend $\tilde{\chi}$ to $\chi$ on $C_{0}$, write the rectangular matrix $M$ whose $n_{c}=\operatorname{rank}\left(B_{0}\right)$ columns are the integral expansions of a minimal basis of $B_{0}$ in a basis of $C_{0} ; M$ has $n_{r}=\operatorname{rank}\left(C_{0}\right) \geqslant n_{c}$ rows. $M$ maps $B_{0}$ to $C_{0}$ by inclusion.

$M^{T} M$ is a $n_{c} \times n_{c}$ matrix; if $M^{T} M$ is not singular, we can form the left inverse of $M$ as $\tilde{M}=\left(M^{T} M\right)^{-1} M^{T}$. To show $M^{T} M$ nonsingular, observe that the columns of $M$ are all independent over the integers, so that we can find a matrix $M^{\prime}$ with $n_{r}$ rows and $n_{r}-n_{c}$ columns, all columns orthogonal to those of $M .^{31}$ The $n_{r} \times n_{r}$ square matrix $A=\left(M M^{\prime}\right)$ is therefore nonsingular. Since $A$ and $A^{T}$ are both nonsingular, so is

$$
A^{T} A=\left(\begin{array}{cc}
M^{T} M & 0 \\
0 & M^{\prime T} M^{\prime}
\end{array}\right),
$$

but since $\operatorname{det}\left(A^{T} A\right) \neq 0$, we must have $\operatorname{det}\left(M^{T} M\right) \neq 0$.

Think of $\tilde{\chi}$ as a row vector of rank $n_{c}$; define $\chi=\tilde{\chi} \tilde{M}$ (row vector of rank $n_{r}$ ). This completes the construction of a linear $\chi$ on all $C_{0}$ such that $\Phi_{g}(\mathbf{k})=\chi(g \mathbf{k}-\mathbf{k})$, proving Theorem 1 . 
${ }^{1}$ D. Shechtman, I. Blech, D. Gratias, and J. W. Cahn, Phys. Rev. Lett. 53, 1951 (1984).

${ }^{2}$ A. Bienenstock and P. P. Ewald, Acta Crystallogr. 15, 1253 (1962)

${ }^{3}$ D. S. Rokhsar, D. C. Wright, and N. D. Mermin, Phys. Rev. B 37, 8145 (1988).

${ }^{4}$ D. A. Rabson, N. D. Mermin, D. S. Rokhsar, and D. C. Wright, Rev. Mod. Phys. 63, 699 (1991).

${ }^{5}$ N. D. Mermin, Rev. Mod. Phys. 64, 3 (1992); 64, 635(E) (1992); 64, 1163(E) (1992); 66, 249(E) (1994).

${ }^{6}$ N. D. Mermin and R. Lifshitz, Acta Crystallogr., Sect. A: Found. Crystallogr. 48, 515 (1992); R. Lifshitz and N. D. Mermin, ibid. 50, 72 (1994); 50, 85 (1994).

${ }^{7}$ R. Lifshitz, Rev. Mod. Phys. 69, 1181 (1997).

${ }^{8}$ N. D. Mermin, Phys. Rev. Lett. 68, 1172 (1992).

${ }^{9}$ N. D. Mermin, Phys. Status Solidi A 151, 275 (1995).

${ }^{10}$ J. Dräger and N. D. Mermin, Phys. Rev. Lett. 76, 1489 (1996).

${ }^{11}$ Henceforth, we shall understand the provision "modulo unity" to apply to all such functions. (Other workers in this field use the symbol $\equiv$ to denote equality modulo 1 .)

${ }^{12}$ N. D. Mermin, D. S. Rokhsar, and D. C. Wright, Phys. Rev. Lett. 58, 2099 (1987). Up to rotation and scale, the standard $2 n$-fold (sub)lattice in the plane is the set of all integral linear combinations of the $2 n$th roots of unity, corresponding to a principal ideal; nonprincipal lattices, possible for almost all $2 n \geqslant 46$, correspond to nonprincipal ideals.

${ }^{13}$ S. R. Renn and T. C. Lubensky, Phys. Rev. A 38, 2132 (1988); T. C. Lubensky, T. Tokihiro, and S. R. Renn, Phys. Rev. Lett. 67, 89 (1991); T. C. Lubensky, S. R. Renn, and T. Tokihiro, in Quasicrystals: The State of the Art, edited by D. P. DiVincenzo and P. J. Steinhardt (World Scientific, Singapore, 1991).

${ }^{14}$ L. Navailles, P. Barios, and H. Nguyen, Phys. Rev. Lett. 71, 545 (1993).

${ }^{15}$ W. M. Itano, J. J. Bollinger, J. N. Tan, B. Jelenković, X.-P. Huang, and D. J. Wineland, Science 279, 686 (1998); J. J. Bollinger, T. B. Mitchell, X.-P. Huang, W. M. Itano, J. N. Tan, B. M. Jelenković, and D. J. Wineland, Phys. Plasmas 7, 7 (2000).

${ }^{16}$ A. König and N. D. Mermin, Phys. Rev. B 56, 13607 (1997); Proc. Natl. Acad. Sci. U.S.A. 96, 3502 (1999); Am. J. Phys. 68, 525 (2000).

${ }^{17}$ The special points $\mathbf{q}$ admitting nontrivial $G_{\mathbf{q}}$ fall into three categories: (1) lattice points, (2) $\mathbf{k}_{g} \neq 0$, which in the periodic case puts $\mathbf{q}$ on a Brillouin-zone boundary, and (3) $\mathbf{k}_{g}=0$, i.e., $\mathbf{q}$ invariant under $g$. The first category is uninteresting because Eq. (6) always vanishes (for commuting $g, h \in G_{\mathbf{q}}$ ) as a consequence of Eq. (4). The third category is similarly uninteresting, since the corresponding phase must vanish.

${ }^{18}$ K. S. Brown, Cohomology of Groups (Springer, New York, 1982).

${ }^{19}$ I. Schur, J. Reine Angew. Math. 127, 20 (1904); reprinted in I. Schur, Gesammelte Abhandlungen (Springer, Berlin, 1973), Vol. 1, p. 86.
${ }^{20}$ A. Janner and T. Janssen [Physica (Amsterdam) 53, 1 (1971)] have discussed the connection between group cohomology and gauge in the context of the Poincare group and electromagnetic potentials.

${ }^{21}$ Conforming to mathematical notation, we use $\operatorname{Hom}(A, B)$ to mean the set of linear functions mapping $A$ into $B, \operatorname{ker}(d)$ to indicate the kernel of a map $d$ (i.e., the set of arguments that $d$ maps to zero), $\operatorname{im}(d)$ for its image [i.e., if $d$ maps $A$ to $B$, that subset of $B$ that can be written as $d(a)$ for some $a \in A]$, R to denote the real numbers, $\mathbb{Z}$ the integers, $Q$ the rationals, and $R / Z$ the interval $[0,1)$.

${ }^{22}$ One convenient formulation of the general rule states

$$
\begin{aligned}
(d \sigma)\left(g_{1}, \ldots, g_{n+1}\right)= & \sigma\left(g_{1}, \ldots, g_{n}\right) g_{n+1} \\
& +(-1) \sigma\left(g_{1}, g_{2}, \ldots, g_{n} g_{n+1}\right)+\cdots \\
& +(-1)^{n} \sigma\left(g_{1} g_{2}, g_{3}, \ldots, g_{n+1}\right) \\
& +(-1)^{n+1} \sigma\left(g_{2}, g_{3}, \ldots, g_{n+1}\right) .
\end{aligned}
$$

By $\sigma(g) h=\sigma(g) \circ h$, one should understand that group element $h$ acts on a member $\mathbf{k}$ of $\mathcal{L}$ before the function $\sigma(g)$ then acts on $h \mathbf{k}$.

${ }^{23}$ The general recipe is

$$
\begin{aligned}
\partial\left(\mathbf{k}\left[g_{1}\left|g_{2}\right| \cdots \mid g_{n}\right]\right)= & \left(g_{n} \mathbf{k}\right)\left[g_{1}\left|g_{2}\right| \cdots \mid g_{n-1}\right]+(-1) \\
& \times \mathbf{k}\left[g_{1}\left|g_{2}\right| \cdots \mid g_{n-1} g_{n}\right]+\cdots+(-1)^{n-1} \\
& \times \mathbf{k}\left[g_{1} g_{2}|\cdots| g_{n}\right]+(-1)^{n} \mathbf{k}\left[g_{2}\left|g_{3}\right| \cdots \mid g_{n}\right] .
\end{aligned}
$$

While this differs from Brown's formula, his duality theorem (7.4) can be seen still to follow by reversing the order of the group elements $g_{i}$ and replacing each with its inverse.

${ }^{24}$ E. Ascher and A. Janner [Commun. Math. Phys. 11, 138 (1968)] state in direct-space language as corollary 2.1.1 the first statement in our Lemma 2 for crystallographic (periodic) groups, ascribing to Schur the method of summing over group elements. For an interpretation of this result in terms of real-space crystallography, see their proposition 5.1.

${ }^{25}$ Brown (Ref. 18), theorem 7.4, p. 147. A "free" Abelian group (represented additively) is one for which no nontrivial integral linear combination of a minimal set of generators vanishes.

${ }^{26}$ N. Jacobson, Basic Algebra I (Freeman, San Francisco, 1974); see Sec. 3.7.

${ }^{27}$ B. Fisher and D. A. Rabson, math-ph/0105010 (unpublished).

${ }^{28}$ The astute reader will notice the absence of a $g_{n+1}$ element acting to the left on the first term. This is because of the trivial group action $\Lambda\left(g_{1}, g_{2}\right) \circ g_{3}=\Lambda\left(g_{1}, g_{2}\right)$.

${ }^{29}$ E. Ascher and A. Janner, Helv. Phys. Acta 38, 551 (1965); T. Janssen, A. Janner, and E. Ascher, Physica (Amsterdam) 42, 41 (1969).

${ }^{30}$ H. Hiller, Am. Math. Monthly 93, 765 (1986).

${ }^{31}$ Since the columns of $M$ are independent over $\mathbb{Z}$, they are independent over Q, which is a field, so we can choose the columns of $M^{\prime}$ as not just independent of those of $M$, but also orthogonal. 